\title{
Analysis of chemokine receptors from angiosarcoma as the potential tumor marker
}

Low-risk method presents quick turnaround of the degenerative disease, researchers found

A

round 35\% of non-metastatic angiosarcoma's survival rate has been reported by researchers to be five years due to its poor prognosis and that it is "a rapidly progressing malignant skin tumor."

"Angiosarcoma is a rare malignant skin tumor and appears as a pink-dark red erythema in the frontal region over the forehead in the beginning and it frequently develops in the elderly" described by Tomoo Kishi and his co-authors from Department of Dermatology, Wakayama Medical University, Japan in an original research article published in the Journal of Surgical Dermatology.

The authors wrote "Angiosarcoma is accompanied by edema and then form erosion, exudative and a crust". At advance stages of angiosarcoma, "nodules with ulceration occur", they added.

According to global medical resource portal Medscape.com, "These tumors have a high local recurrence rate and metastasis because of their intrinsic biologic properties and because they are often misdiagnosed, leading to a poor prognosis and a high mortality rate."

In addition, according to the global sarcoma advocate
Liddy Shriver Sarcoma Initiative, patients are usually diagnosed with angiosarcoma at a later stage where the disease has spread throughout the body. Therefore, it is a need to find a better solution to overcome this poor prognosis problem.

The presence of cancer is detected by the help of tumor markers. As explained by National Cancer Institute, tumor markers are made by both normal and cancer cells, but the production of tumor markers are higher in cancerous conditions. Most of the tumor markers are proteins such as chemokines that can be found in the blood, tumor tissue and body fluid of some cancer patients.

"Chemokines are substances inducing the migration of specific white blood cells and lymphocytes, and it has been reported that chemokine and chemokine receptor expressions play important roles in the outgrowth, infiltration, and metastasis of malignant tumors" authors described.

The authors also added that chemokines represents cytokine proteins with chemotactic activity and, in the late 1980s, the study of chemokines started with the discovery of a factor inducing neutrophil migration and activation, interleukin (IL)-8. Subsequently, studies since then have greatly advanced and "more than 50 chemokines

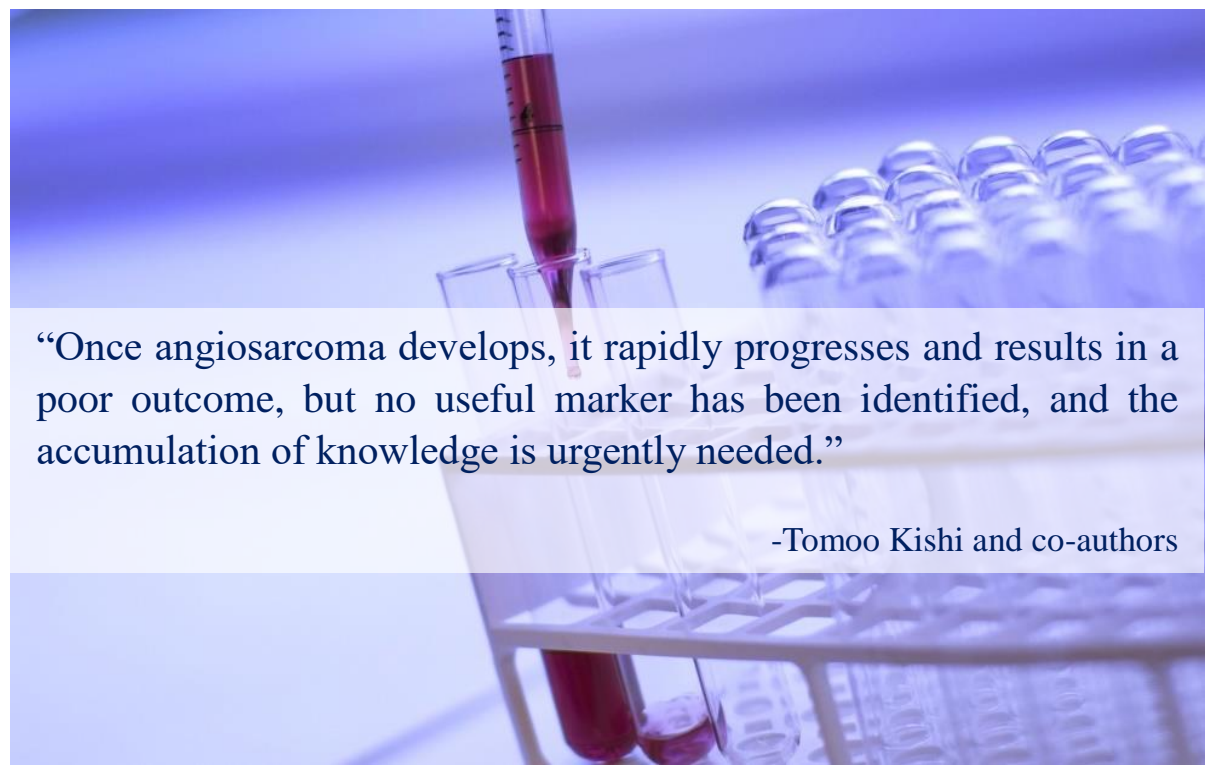

Image credit: Philippe Delavie via Pixabay 
have been identified."

In a previous investigation done by the authors on expressions of CXCR4, CCR6, CCR7 and SDF-1 in malignant melanoma, they initially found that CXCR4 was a useful marker for malignancy.

As explained by the authors, "CXCR4 is a specific receptor of the chemokine SDF-1, and it is expressed on the cell membrane of white blood cells and undifferentiated hematopoietic cells."

Nevertheless, the authors claimed that an analysis of chemokines and chemokine receptors in angiosarcoma has not been performed yet. No useful marker has yet been identified, added Tomoo Kishi and his co-authors.

Using 12 tumor tissue samples of angiosarcoma from patients of Wakayama Medical University Hospital, the authors aimed to investigate chemokine receptor expression, and if it is expressed, to histologically discuss its association with the age, sex, development site, and histologic type. The tumor tissues were collected via biopsy or surgery from patients with mean age of 75.7 years old. As a control, "16 healthy skin samples were analyzed," explained the authors.

From their investigation, they found out that CXCR4 expression was positive in 6 of the 12 samples. Among of those 6 samples, there were 4 cellular rich type and 2 were vascular rich type. In addition, they reported that the expression of CCR6 and CCR7 were negative in all samples.
The authors also mentioned that the expression of CXCR4/SDF-1 was slightly higher in malignant melanoma when a tumor thickness of $2 \mathrm{~mm}$ or less, histological classification of the node or non-node type, and the presence or absence of distant metastasis was within two years.

Besides that, the authors also investigated the association of the expressed chemokines with demographic parameters such as age, sex, development site and histologic type. They found out that there is no significant difference associated with the age, sex, development site or histologic type in the expression of either CXCR4, CCR6, CCR7, or SDF-1.

Finally, the authors claimed that, although CXCR4 is regarded as an index of tumor aggressiveness of malignant melanoma, it was suggested that chemokine receptors, such as CXCR4, do not serve as a useful marker of angiosarcoma.

Their research ultimately highlights the need for further investigation on the usability of these chemokines as a marker as a tumor marker for angiosarcoma. "We have to collect more cases of angiosarcoma and analyze them immunohistochemically," they write. "More development of a new chemokine and tumor marker shall be needed in this disease," the authors concluded.

Yuki Yamamoto, Chikako Kaminaka, Seiko Toyozawa, Hiroshi Matsunaka, and Fukumi Furukawa are co-authors of this featured research article which is published on pages 5-11 in this JSD issue. 